The median of the first ultrasound examination of children from the DCS group was 75 days (min 30-max 210 days), children from CG 77.5 days (min 30-max 210 days).

Kidney lengths according to age, gender and side in infants from the DCS group were compared with kidney length from CG and with kidneys from CDCS group as shown in the eight dot graphs.

Of the 38 kidneys from the DCS group, 23 (60\%) were above the upper limit of $95 \%$ CI for age, gender and side $(\chi 2=22.6 ; \mathrm{p}<0.001)$.

Of the 28 kidneys from the CDCS group, one (3.6\%) was above 95\% CI in length, and in the CG of 1896 kidneys, 59 (3.1\%) were above 95\% CI in length $(\chi 2=0.02 ; \mathrm{p}=$ 0.889 ). By comparing the CG and the DCS group, the statistically significant difference was obtained $(\chi 2=302 ; \mathrm{p}$ $<0.001)$ in terms of longer kidney length with DCS.

In the group of infants with DCS there are 20 times more kidneys that are above the upper limit of $95 \%$ CI in length for age, gender and side in relation to CG and 17 times more than in CDCS.

Conclusion We found that kidneys with DCS were statistically significantly longer than kidneys with one collecting system positioned contra laterally to kidney with DCS, also longer than kidneys in CG of the same age group of children.

Our research proved that nothing significant happens to the kidney contralateral to the kidney with DCS, which would affect its growth. When a longer than normal kidney is found on ultrasound, it is necessary to analyse it in detail in terms of detecting DCS and possibly associated urinary tract anomalies.

\section{NEPHROCALCINOSIS, HYPOMAGNESEMIA AND HYPERCALCIURIA IN BROTHER AND SISTER: ARE GENES DESTINY?}

Ivan Jakopčić ${ }^{*}$, Hana Matković, Ivanka Kos, Lovro Lamot, Kristina Vrljičak. Department of Pediatrics, University Hospital Centre Zagreb, University of Zagreb School of Medicine, Zagreb, Croatia

\subsection{6/archdischild-2021-europaediatrics.384}

Introduction Nephrocalcinosis is a condition characterized by calcium deposition in the renal parenchyma that can lead to a renal failure. Typically, it is not accompanied by specific symptoms, but lesser number of children might develop a recurrent urinary tract infection (rUTI). Therefore, in children with rUTI nephrocalcinosis should be considered as a possible underlying cause, prompting additional imaging and laboratory diagnostics, and depending on the findings, in some cases even genetic testing.

Case Report We report the case of an eleven-year-old girl and her seven-year-old brother with nephrocalcinosis. The girl initially presented at the age of four with recurrent urinary tract infections and bilateral nephrocalcinosis detected by renal ultrasound. During the further evaluation hypercalciuria, hypomagnesaemia, hypermagnesiuria, hyperparathyroidism, hyperuricemia, decreased glomerular filtration and bilateral optic neuropathy were detected. Since hereditary disease was suspected, her younger brother underwent a renal ultrasound at the age of 4 months with the similar finding of bilateral nephrocalcinosis. Moreover, laboratory testing indicated the presence of same abnormalities as in his sister, but in the absence of other clinical symptoms. Finally, targeted gene sequencing in both siblings (Blueprint Nephrolitiasis panel with 35 genes) identified in CLDN16 gene a novel heterozygous frameshift variant c.332_333del, p.(Thr111Lysfs*15) previously not described, as well as heterozygous missense variant c.358T >C, p.(Cys120Arg) previously described in one person. Both variants are classified by in silico methods as pathogenic, while sequencing data strongly suggests that they are on different parenteral alleles (in trans position).

Discussion Pathogenic variants of the CLDN16 gene cause renal hypomagnesaemia, an autosomal recessive tubulopathy also known as familial hypomagnesaemia with hypercalciuria and nephrocalcinosis (FHHNC). This disease is characterized by excessive loss of magnesium and calcium in the urine, polyuria, polydipsia, bilateral nephrocalcinosis and progressive chronic renal failure. It can also affect the eye and present with symptoms such as strabismus, nystagmus, hyperopia, myopia and astigmatism. Biochemical findings characteristic of the disease include hyperuricemia, hypermagnesiuria, hypercalciuria, hypocitraturia, hematuria and leukocyturia, often without bacteriuria. The onset of the disease is usually in the childhood or adolescence. Magnesium citrate as a replacement therapy in combination with thiazide diuretics is most commonly used for treatment, although in the advanced stage of the disease kidney transplantation remains the only viable option. In the presented case of siblings, despite the same complex heterozygous variants previously undescribed in the literature, there are marked differences in clinical phenotype, demonstrating a significant role of other, external factors in the development of the disease. Nevertheless, in all patients with nephrocalcinosis, it is important to consider genetic testing in order to confirm underlying monogenic diseases, determine the risk in other family members, select the most appropriate treatment options and predict the possible course.

\section{Paediatric Neurology}

\section{5 CONGENITAL CYTOMEGALOVIRUS INFECTION OR GENETIC SYNDROME? A TWO-CASE-REPORT COMPARISON}

Mara Fumagalli*, Laura Gianolio, Vania Giacomet, Gian Vincenzo Zuccotti. Department of Pediatric, Vittore Buzzi Children's Hospital

10.1136/archdischild-2021-europaediatrics.385

Congenital cytomegalovirus infection (cCMV) is the most common congenital viral infection; however, a poor rate of awareness is reported worldwide. Congenital cytomegalovirus' most frequent and severe expression is represented by neuroaudiological impairment. We report two cases of late diagnosed cCMV associated with severe neuro-radiological alterations.

Case-1: a 14-month-old infant presented with left upper limb weakness and tendon hyperreflexia. Slight dysmorphic features (frontal bossing and low-set ears) were observed. Brain magnetic resonance imaging revealed cortical dysplasia and wallerian degeneration of right-corticospinal tract.

CVM-DNA detection on Guthrie-card resulted positive and no anomalies were found at whole-exome-sequencing.

Case-2: a 3-month-old infant presented with focal epilepsy, developmental delay and dysphagia. Audiological assessment revealed left severe hearing loss. At brain magnetic resonance imaging fronto-temporal polymicrogyria was found. CVM- 
DNA detection on Guthrie-card resulted positive and a novel gene mutation in PLP1 was found at whole-exome-sequencing.

In both cases neurological impairment differential diagnosis between $\mathrm{cCMV}$ and genetic syndrome appeared particularly challenging. bMRI suggested for both a cCMV causative role: in case- 1 radiologic imaging revision revealed a possible fetal clastic damage, in case- 2 grey matter degeneration with normal white matter appeared inconsistent with PLP1 mutation. Moreover, a detailed perinatal history revealed early signs of cCMV, which were ignored: in case-1 intrauterine-growthrestriction, in case-2 pathological audiological screening.

In conclusion, congenital cytomegalovirus infection should always be considered in cases of infant with unexplained neurological impairment, particularly when signs suggestive of congenital infection are present at birth. In fact, an early screening of possible neurological sequelae allows precocious treatment whose efficacy is documented only if started within the first month of life.

\section{EPILEPSY IN PEDIATRIC PATIENTS - EVALUATION OF ANATOMIC STRUCTURES' VOLUME OF THE BRAIN}

Ewa Kopyto*, Małgorzata Matuszek, Olga Pustelniak, Katarzyna Drelich, Weronika Cyranka, Monika Zbroja, Andrzej Materniak, Magdalena Woźniak. Students' Scientific Society at the Department of Pediatric Radiology, Medical University of Lublin

\subsection{6/archdischild-2021-europaediatrics.386}

Epilepsy is a disease of the central nervous system with somatic, vegetative and psychological symptoms that appear as a result of various morphological and metabolic changes in the brain. Epileptic seizures are the manifestation of temporary problems with communication between nerve cells. About 1\% of the world's population suffers from epilepsy. Published articles have focused so far on evaluating changes in adult patients. The aim of the study was to evaluate anatomic structures' volume of the brain in pediatric patients with epilepsy.

A group of 42 pediatric patients with clinical symptoms of epilepsy (study group) and 16 healthy patients (experimental group) aged 3 months-17 years were enrolled in the study. Brain MR imaging was performed in all children according to a dedicated protocol (epilepsy specific protocol). Individual anatomical structures of the central nervous system were analyzed on the basis of T1-wieghted 3D isometric $1 \mathrm{~mm}$ sequence and volume changes of specific structures were compared between the epilepsy group and the control group.

In the study group, the ratio of brain tissue to CSF was $89,08 \%$ to $10,92 \%$, while in the control group it was $90,99 \%$ to $9,01 \%$, In the research group compared to the control group, the volumes of each brain structure were: cerebrum $77,99 \% / 79,68 \%$, cerebellum - 9,65\%/9,87\%, brainstem $1,41 \% / 1,44 \%$, lateral ventricle - 1,41\%/0,49\%, caudate $0,54 \% / 0,55 \%$, putamen - 0,61\%/0,68\%, thalamus - 0,82\%/ $0,91 \%$, globus pallidus - 0,18\%/0,19\%, hipoccampus $0,45 \% / 0,50 \%$, amygdala - 0,11\%/0,10\%, accumbens - 0,04\%/ $0,05 \%$.

During the course of epilepsy in pediatric patients, there is a decrease in the volume of brain tissue, with particular emphasis on the cerebrum, cerebellum, brainstem, caudate, putamen, thalamus, globus pallidus, hippocampus and accumbens, moreover an increase in the volume of lateral ventricles. The study indicates cortical and subcortical atrophy in pediatric patients with epilepsy. The data obtained have important clinical and prognostic significance, however they need to be confirmed on a large study group with taking into account changes in the volume of anatomical structures of the brain in relation to age and disease duration

\section{EVOLUTION OF FOCAL EPILEPTIFORM DISCHARGES IN THE ELECTROENCEPHALOGRAPHY OF THE PREMATURE NEWBORN WITH WHITE MATTER LESIONS}

Branka Bunoza*, Nina Barišić. University Hospital Centre Zagreb

\subsection{6/archdischild-2021-europaediatrics.387}

Advances in neonatal medicine has dramatically improved survival rate of preterm infants that are frequently born with numerous neurodevelopmental disabilities including epileptic seizures and epilepsy. In this dissertation the hypothesis was that localization of white matter injury is associated with epileptiform EEG changes and motor development.

In 64 preterm infants we analyzed EEG, brain MRI and ultrasound and motor development We evaluated the relationship of brain white matter lesion with EEG and lesions of certain brain localization (segment II - crossroads than parietal, temporal, occipital lobe, basal ganglia, cerebellum and ventriculomegaly) with epileptic seizures and neurodevelopmental outcome.

The total number of white matter lesions was in a positive correlation with the pathological EEG findings and epileptic seizures in infants. Infants who did not have visible anterior and posterior crossroads frequently had epileptic seizures during newborn period and worse neurodevelopmental outcome.

The obtained results indicate that white matter damage is associated with epileptogenesis. Among individual localization, the visibility of segment II as the place where commissural, projection and associative fibers are crossing has a prognostic value for the future neurodevelopmental outcome.

Further studies are needed in order to determine the pathogenic factors that contribute to epileptogenesis in infants with white matter lesions

\section{HSV-1 ENCEPHALITIS MIMICKING BILATERAL MCA STROKES IN A YOUNG TODDLER}

Maymunah Khries*, Ines Roncero, Jan Bengtsson. Great North Children's Hospital, Newcastle

\subsection{6/archdischild-2021-europaediatrics.388}

A 15-month-old girl presented with a 2-day history of altered behaviour, lethargy and vomiting. She developed a focal left motor seizure that responded to buccal midazolam. She was encephalopathic and apyrexial at presentation but had fever while in the ward. Initial biochemical investigations were not suggestive of infective or metabolic conditions. A CT angiogram and an MRI head showed evidence of multifocal infarct within the middle cerebral artery territories bilaterally and a small area of haemorrhagic transformation on the right frontal region. There were also older bilateral thalamic infarct noted. Echocardiography study was normal. There was no evidence of thrombus from any of the imaging modalities.

Cefotaxime, aciclovir and low-molecular-weight heparin were commenced early. 